\title{
Calidad del agua de escorrentía para uso agrícola captada en bordos de almacenamiento
}

\section{Runoff water quality for agricultural use captured on earthen dams}

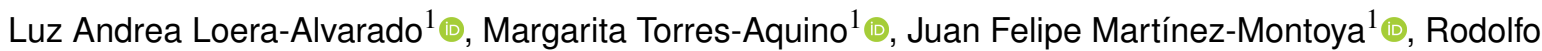 \\ Cisneros-Almazán²], José de Jesús Martínez-Hernández ${ }^{1 *}$ *0
}

\footnotetext{
${ }^{1}$ Colegio de Postgraduados, Campus San Luis Potosí. Iturbide No. 73, CP. 78620. Salinas de Hidalgo, San Luis Potosí. México. ${ }^{2}$ Universidad Autónoma de San Luis Potosí. Facultad de Ingeniería. Av. Dr. Nava No. 8, Zona Universitaria. CP. 78290. San Luis Potosí, México.

*Autor de correspondencia: jjesus@colpos.mx
}

Artículo científico recibido: 29 de mayo de 2018 aceptado: 05 de marzo de 2019

RESUMEN. En las zonas áridas y semiáridas el agua es uno de los factores críticos para la productividad agrícola y pecuaria. La escasez del recurso y el abatimiento de acuíferos son problemas específicos de estas regiones donde la captación de agua de lluvia se vuelve indispensable; sin embargo, no existe información sobre su calidad. Por lo anterior, el objetivo fue determinar la calidad y la aptitud del agua de lluvia captada en bordos de almacenamiento (BA) para su uso en el riego agrícola. Se tomaron muestras de agua de BA ubicados en tres microrregiones del Estado de San Luis Potosí, en las que se determinó el pH, $\mathrm{CE}\left(\mathrm{dS} \mathrm{m}{ }^{-1}\right.$ ), aniones y cationes. Además, se estimaron los índices de Relación de Adsorción de Sodio (RAS), Carbonato sódico residual (CSR), Porcentaje de sodio posible (PSP), Índice de permeabilidad (IP), Índice de Kelly (IK), Dureza, RASaj, Sólidos disueltos totales (SDT), Salinidad efectiva (SE) y Salinidad potencial (SP). Con excepción del magnesio, no hubo diferencias significativas entre microrregiones $(p>0.05)$. De acuerdo con la clasificación de Wilcox, normas de Riverside y valores de IP, IK, SDT, SE y SP, el agua de BA se clasifica como de buena calidad y es apropiada para riego con potencial para la producción de forraje.

Palabras clave: Composición química, cultivos forrajeros, irrigación, reservorios, salinidad.

ABSTRACT. In arid and semi-arid areas, water is one of the critical factors for agricultural and livestock productivity. The shortage of the resource and the depletion of aquifers are specific problems of these regions where the collection of rainwater becomes essential, however, there is not information about its quality. Therefore the objective was to determine the quality and suitability of rainwater collected on earthen dams (ED) for use in agricultural irrigation. Water samples were collected from ED located in three micro-regions of the State of San Luis Potosí, where $\mathrm{pH}, \mathrm{EC}\left(\mathrm{dS} \mathrm{m} \mathrm{m}^{-1}\right.$ ), anions and cations were determined. In addition, Sodium adsorption ratio (SAR), Residual sodium carbonate (RSC), Percentage of possible sodium (PPS), Permeability index (PI), Kelly index (KI), Hardness, SARaj, Total dissolved solids (TDS), Effective salinity (ES) and Potential salinity (PS) were estimated. With the exception of magnesium, there were not significant differences between the micro-regions ( $p>0.05$ ). According to the Wilcox classification, Riverside standards and values of PI, KI, TDS, ES y PS, the water of ED is classified as of good quality and is appropriate for irrigation with potential for the production of forage.

Key words: Chemical composition, forage crops, irrigation, reservoirs, salinity.

\section{INTRODUCCIÓN}

En las regiones áridas y semiáridas de México, el principal factor limitante para la agricultura es el agua, debido a que es indispensable para el crecimiento y desarrollo de las plantas. La FAO (2013) señala que el uso de agua se incrementó a nivel mundial a un ritmo del doble del aumento de la población en el siglo XX y la agricultura es el sector económico que más demanda agua. La irrigación para la producción agrícola depende en gran medida del agua subterránea, lo cual reduce sus niveles de manera alarmante (Pimentel et al. 2004). En el 2017, la Comisión Nacional del Agua publicó la 
disponibilidad de agua de los 653 acuíferos del país. De estos, 32 están en suelos salinos, 18 tienen intrusión de agua marina y 105 están sobreexplotados (CONAGUA 2018). Bajo este contexto, en las regiones áridas y semiáridas, la recolecta y almacenamiento de agua de lluvia es importante para el manejo sostenible del recurso hídrico (Rosegrant et al. 2002).

La recolección de agua de lluvia, es una tecnología en la que la escorrentía superficial es recolectada de forma eficaz (Helmreich y Horn 2009). Por lo que es una alternativa viable para suministrar agua en los hogares, consumo animal y la irrigación de cultivos forrajeros (Sazakli et al. 2007, Helmreich y Horn 2009). Diversos estudios demuestran que la cosecha de agua de lluvia es una opción para producir cultivos bajo riego deficitario, como lo indican Kahinda et al. (2007), Oweis y Hachum (2006) y Akhtar et al. (2016), quienes encontraron incremento de la productividad del agua. Se tienen reportes de la calidad del agua de lluvia en países industrializados, pero se tiene poca información para zonas rurales (Klaus-Dieter 2008). La mayoría de los trabajos realizados sobre cosecha de agua analizan la composición química del agua de lluvia para consumo humano y doméstico (Lee et al. 2010, Cobbina et al. 2013, Mahato et al. 2016). Pero se carece de información sobre la calidad del agua de lluvia para su uso en el riego agrícola. Por lo anterior, el objetivo del presente trabajo fue determinar la calidad y la aptitud del agua de lluvia captada en bordos de almacenamiento (BA) para la irrigación de cultivos forrajeros en tres microrregiones de San Luis Potosí.

\section{MATERIALES Y MÉTODOS}

\section{Área de estudio}

La investigación se llevó a cabo en tres municipios del Estado de San Luis Potosí, localizados en las microrregiones económicas: Altiplano Centro (MAC), Centro Sur (MCS) y Media Oeste (MMO). Los sitios tienen clima semiseco con vegetación predominante de pastizal y matorral xerófilo (INEGI 2011). La precipitación promedio anual varía entre 600 y $700 \mathrm{~mm}$ en MMO (Rioverde), mientras que en la MAC (Ve- nado) y MCS (Villa de Reyes) es de $400 \mathrm{~mm}$ (INEGI 2006). El tipo de suelo, en Rioverde (MMO) es litosol y feozem, que son suelos delgados con poca profundidad (litosol) y suelos ricos en materia orgánica, rocosos y erosionables (feozem), este último también predomina en Villa de Reyes (MCS) mientras que en Venado (MAC) son los cambisoles, suelos jóvenes poco desarrollados, con capa mayor de $15 \mathrm{~cm}$ de espesor, enriquecida con carbonatos secundarios (SEMARNAT 2015).

\section{Muestreo y análisis de agua}

Se seleccionaron tres BA por microrregión, los cuales se ubicaron en un mapa digital empleando el programa ArcGIS 10.3 (Figura 1). Los BA del Bajío de la Gallina y El Botón localizados en el municipio de Venado, así como el BA Agua Nueva en Rioverde, son obras con cortina de tierra compactada y vertedor de mampostería. Mientras que los BA restantes, además de los elementos ya mencionados, tienen un cerco perimetral, presas filtrantes y un bebedero pecuario. A partir de cartas topográficas específicas, datos vectoriales (escala 1:50 000) serie III (INEGI 2016) para trazar las rutas de acceso a cada uno de los BA, para luego realizar un recorrido. De junio a noviembre del 2016, en cada bordo se tomaron las muestras de agua de acuerdo con la NOM-230SSA1-2002 (SSA 2005). La determinación de pH y conductividad eléctrica ( $\mathrm{dS} \mathrm{m}^{-1}$ ) se realizó in situ con el potenciómetro 850051 y conductímetro 850037 de Sper Scientific, respectivamente. En laboratorio, se determinó la concentración de potasio $\left(\mathrm{K}^{+}\right)$y sodio $\left(\mathrm{Na}^{+}\right)$con el fotómetro de llama (Instrumentation Laboratory ${ }^{\circledR}$ Auto Cal Flame Photometer 643). Los carbonatos $\left(\mathrm{CO}_{3}^{2-}\right)$ y bicarbonatos $\left(\mathrm{HCO}_{3}^{-}\right)$se obtuvieron por titulación volumétrica con ácido sulfúrico $0.1 \mathrm{~N}$. La concentración de cloruros $\left(\mathrm{Cl}^{-}\right)$se determinó por titulación con nitrato de plata $0.01 \mathrm{~N}$ y para determinar el calcio $\left(\mathrm{Ca}^{2+}\right)$ y magnesio $\left(\mathrm{Mg}^{2+}\right)$ se utilizó EDTA $0.01 \mathrm{~N}$. Los sulfatos $\left(\mathrm{SO}_{4}^{2-}\right)$ se analizaron por espectrofotometría a $420 \mathrm{~nm}$ (Jenway 7305 Spectrophotometer).

\section{Aptitud del agua para su uso en irrigación}

Para determinar la calidad del agua de los BA 


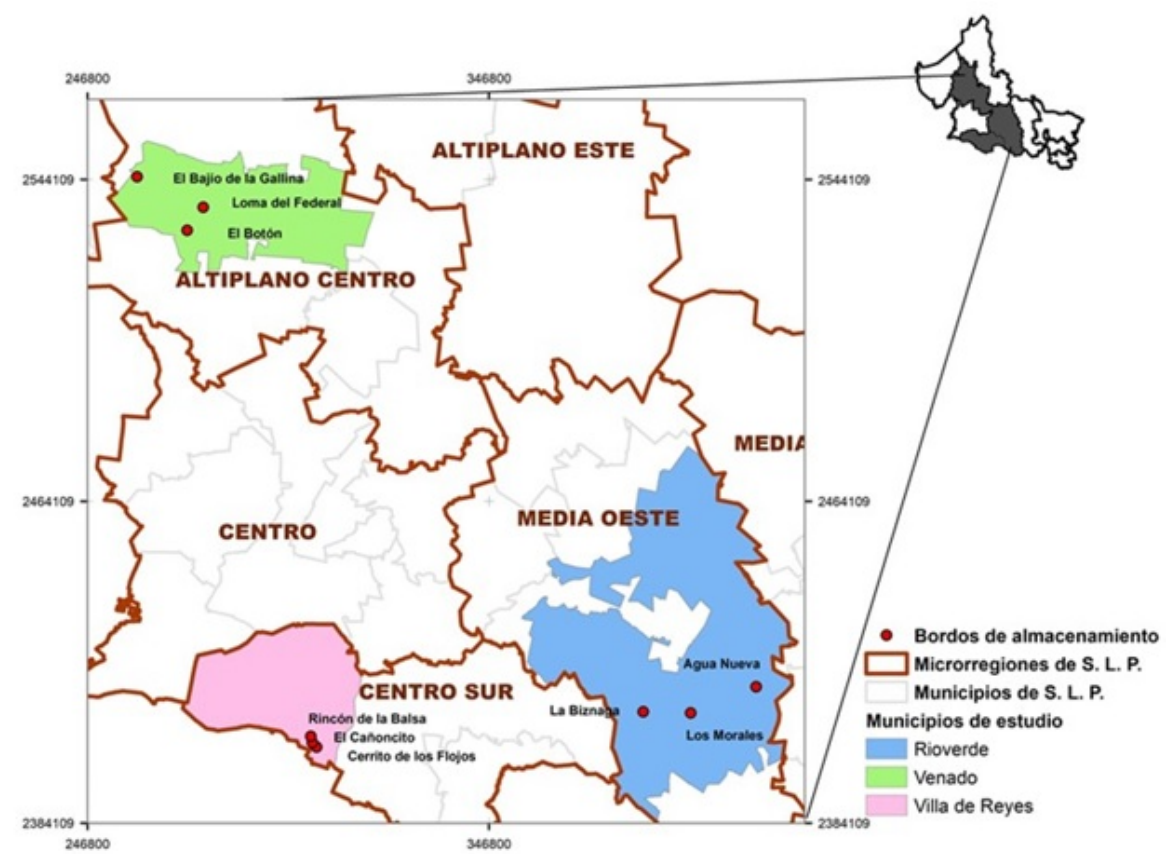

Figura 1. Ubicación de bordos de almacenamiento de estudio en tres microrregiones de San Luis Potosí.

se utilizó la Clasificación de Wilcox (1948) que relaciona la CE con el porcentaje de sodio con respecto al total de cationes. Se utilizaron las Normas de Riverside (Richards 1954), que emplean la conductividad eléctrica (CE) y la Relación de Adsorción de Sodio (RAS) para clasificar el agua de riego. Además se calcularon los índices RAS, CSR, PSP, IP, IK, Dureza expresada en grados hidrotimétricos franceses $\left({ }^{\circ} \mathrm{fH}\right)$, RASaj, SDT, SE y SP (Tabla 1) para determinar la aptitud del agua del BA. Para conocer el potencial del agua del BA sobre el rendimiento relativo de los cultivos forrajeros: avena, maíz, cebada y sorgo, representativos de la producción de forraje en el Estado de San Luis Potosí (SEDUCOP 2012) se determinó la conductividad del extracto de saturación del suelo con la CE del agua (García 2012).

\section{Análisis estadístico}

A las variables de respuesta: $\mathrm{pH}$ del agua, CE, concentraciones de aniones $\left(\mathrm{CO}_{3}^{2-}, \mathrm{HCO}_{3}^{-}, \mathrm{SO}_{4}^{2-} \mathrm{y}\right.$ $\left.\mathrm{Cl}^{-}\right)$, cationes $\left(\mathrm{Ca}^{2+}, \mathrm{Mg}^{2+}, \mathrm{Na}^{+}\right.$y $\left.\mathrm{K}^{+}\right)$e índices estimados, se les aplicó un análisis de varianza y prueba de rango múltiple de Tukey $(p=0.05)$, con el programa Infostat.

\section{RESULTADOS}

\section{Análisis de agua}

Los valores promedio de $\mathrm{pH}$ y $\mathrm{CE}$ no tuvieron diferencias entre microrregiones $(p=0.6687$ y $p=$ 0.3230 , respectivamente). El $100 \%$ de los BA en las tres microrregiones presentaron valores de $\mathrm{pH}$ entre 7.0 y 7.8 con CE entre 0.103 y $0.373 \mathrm{dS} \mathrm{m}^{-1}$ (Tabla 2). En ningún $\mathrm{BA}$ se detectó la presencia de $\mathrm{CO}_{3}^{2-}$. En las tres microrregiones, el anión dominante fue el bicarbonato, con concentración entre 54.9 y 183.0 ppm, seguido por los cloruros (10.6 a 28.4 ppm) y sulfatos (1.4 a 12.0 ppm) sin diferencias significativas entre microrregiones. El catión de mayor concentración en el agua fue el $\mathrm{Ca}^{2+}$ (12.0 a $44.1 \mathrm{ppm}$ ) seguido por el $\mathrm{K}^{+}$(3.5 a $\left.25.0 \mathrm{ppm}\right), \mathrm{Mg}^{2+}$ (4.9 a $12.2 \mathrm{ppm})$ y $\mathrm{Na}^{+}$(0.0 a $\left.9.2 \mathrm{ppm}\right)$ en las microrregiones MCS y MMO. Mientras que en MAC el orden fue $\mathrm{Ca}^{2+}$ (34.1 a $\left.44.1 \mathrm{ppm}\right), \mathrm{K}^{+}$(3.5 a $\left.25 \mathrm{ppm}\right), \mathrm{Na}^{+}$ (0.0 a 18.4 ppm) y $\mathrm{Mg}^{2+}$ (3.6 a 7.3 ppm). Sólo se detectaron diferencias significativas para $\mathrm{Mg}^{2+}(\mathrm{p}=$ 
Tabla 1. Ecuaciones utilizadas para estimar la aptitud del agua para riego.

\begin{tabular}{|c|c|}
\hline Índice & Ecuación \\
\hline Relación de Adsorción de Sodio (Richards 1954) & $R A S=\frac{N a^{+}}{\sqrt{\frac{C a^{2+}+M g^{2+}}{2}}}$ \\
\hline Carbonato de Sodio Residual (Eaton 1950) & $C S R=\left(\mathrm{CO}_{3}^{2-}+\mathrm{HCO}_{3}^{-}\right)-\left(\mathrm{Ca}^{2+}+\mathrm{Mg}^{2+}\right)$ \\
\hline Porcentaje de Sodio Posible (Palacios y Aceves 1970) & $P S P=\frac{N a^{+}}{S E} \times 100$ \\
\hline Índice de Permeabilidad (Doneen 1964) & $I P=\frac{\left(\mathrm{NA}^{+}+\sqrt{\mathrm{HCO}_{3}^{-}}\right) \times 100}{\mathrm{Ca}^{2+}+\mathrm{Mg}^{2+}+\mathrm{Na}^{+}}$ \\
\hline Índice de Kelly (Kelly 1951) & $I K=\frac{\mathrm{Ca}^{2+}}{\mathrm{Ca}^{2+}+\mathrm{Mg}^{2+}+\mathrm{Na}^{+}} \times 100$ \\
\hline Dureza ( ${ }^{\circ}$ franceses) (Vásquez 2000) & ${ }^{\circ} f=\frac{\frac{m g}{L} \mathrm{Ca}^{2+}+\frac{m g}{L} \mathrm{Mg}^{2+}+4.12}{10}$ \\
\hline RAS ajustada (Bower y Wilcox 1965) & $\begin{array}{c}R A S a j=S A R *[1+(8.4-p H c)] \text { donde }: \\
p H c=\left(P k^{\prime} 2-P k^{\prime} c\right)+p\left(C a^{2+}+M g^{2+}\right)+p(A l k)\end{array}$ \\
\hline Sólidos Disueltos Totales (Sparks 1995) & $S D T\left(m g L^{-1}\right)=0.64 * C E\left(\mu S \mathrm{~cm}^{-1}\right)$ \\
\hline Salinidad Efectiva (Doneen 1975) & $\begin{array}{l}S E=\text { suma de cationes }^{*}-\left(\mathrm{CO}_{3}^{2-}+\mathrm{HCO}_{3}^{-}\right) \\
S E=\text { suma de cationes }{ }^{*}-\left(\mathrm{Ca}^{2+}+\mathrm{Mg}^{2+}\right)\end{array}$ \\
\hline Salinidad Potencial (Doneen 1975) & $S P=\mathrm{Cl}^{-}+1 / 2 \mathrm{SO}_{4}^{2-}$ \\
\hline
\end{tabular}

${ }^{*}$ Si la suma de cationes es menor que la suma de aniones, se utiliza la suma de aniones en lugar de la de cationes.

Tabla 2. Parámetros químicos obtenidos en muestras de agua de BA en tres microrregiones de San Luis Potosí.

\begin{tabular}{|c|c|c|c|c|c|c|c|c|c|c|}
\hline \multirow[t]{2}{*}{ Microrregión } & \multirow{2}{*}{$\begin{array}{c}\text { Bordo de } \\
\text { almacenamiento }\end{array}$} & \multirow[t]{2}{*}{$\mathrm{pH}$} & \multirow{2}{*}{$\begin{array}{c}C E \\
\left(d S m^{-1}\right)\end{array}$} & \multicolumn{3}{|c|}{ Aniones (ppm) } & \multicolumn{4}{|c|}{ Cationes (ppm) } \\
\hline & & & & $\mathrm{HCO}_{3}^{-}$ & $\mathrm{Cl}^{-}$ & $\mathrm{SO}_{4}^{2-}$ & $\mathrm{Ca}^{2+}$ & $\mathrm{Mg}^{2+}$ & $\mathrm{Na}^{+}$ & $\mathrm{K}^{+}$ \\
\hline & Bajío de la Gallina & 7.5 & 0.373 & 183 & 28.4 & 10.6 & 36.1 & 7.3 & 18.4 & 25.0 \\
\hline \multirow[t]{4}{*}{ Altiplano Centro } & El Botón & 7.8 & 0.267 & 140.3 & 17.7 & 6.7 & 44.1 & 4.9 & 6.9 & 3.5 \\
\hline & Loma del Federal & 7.6 & 0.223 & 122.0 & 14.2 & 6.3 & 34.1 & 3.6 & 0.0 & 7.0 \\
\hline & Promedio & $7.6^{a}$ & $0.288^{a}$ & $148.5^{a}$ & $20.1^{a}$ & $7.9^{a}$ & $38.1^{a}$ & $5.3^{a}$ & $8.4^{a}$ & $11.9^{a}$ \\
\hline & Cerrito de los Flojos & 7.2 & 0.288 & 134.2 & 17.7 & 9.1 & 36.1 & 8.5 & 4.6 & 18.8 \\
\hline \multirow[t]{4}{*}{ Centro Sur } & Rincón de la Blasa & 7.7 & 0.192 & 85.4 & 14.2 & 10.1 & 24.0 & 8.5 & 4.6 & 15.6 \\
\hline & El Cañoncito & 7.0 & 0.103 & 54.9 & 14.2 & 12.0 & 12.0 & 12.2 & 0.0 & 9.0 \\
\hline & Promedio & $7.3^{a}$ & $0.194^{a}$ & $91.5^{a}$ & $15.4^{a}$ & $10.4^{a}$ & $24.0^{a}$ & $9.7^{b}$ & $3.1^{a}$ & $14.5^{a}$ \\
\hline & La Biznaga & 7.6 & 0.202 & 97.6 & 14.2 & 7.7 & 22.0 & 6.1 & 9.2 & 9.0 \\
\hline \multirow[t]{4}{*}{ Media Oeste } & Los Morales & 7.0 & 0.179 & 85.4 & 10.6 & 7.7 & 20.0 & 6.1 & 0.0 & 8.2 \\
\hline & Agua nueva & 7.5 & 0.258 & 152.5 & 14.2 & 1.4 & 44.1 & 4.9 & 0.0 & 10.2 \\
\hline & Promedio & $7.4^{a}$ & $0.213^{a}$ & $111.9^{a}$ & $13.0^{a}$ & $5.6^{a}$ & $28.7^{a}$ & $5.7^{a b}$ & $3.1^{a}$ & $9.1^{a}$ \\
\hline & Probabilidad de F & 0.4654 & 0.323 & 0.2237 & 0.2342 & 0.162 & 0.3374 & 0.0312 & 0.5287 & 0.6844 \\
\hline
\end{tabular}

Medias con una letra común no son significativamente diferentes $(p>0.05)$.

0.0312), presentando el agua de los BA de la MCS los mayores valores.

\section{Aptitud del agua para su uso en irrigación}

De acuerdo con la clasificación de Wilcox el $100 \%$ de los BA tuvieron agua de excelente a buena calidad (Figura 2). El $55 \%$ de los BA tienen agua $\mathrm{C}_{1} \mathrm{~S}_{1}$ (baja salinidad y baja en sodio) de acuerdo con las Normas de Riverside, mientras que el resto de los BA (El Bajío de la Gallina, El Botón, Cerrito de los Flojos y Agua nueva) tuvieron agua $\mathrm{C}_{2} \mathrm{~S}_{1}$ (salinidad media y baja en sodio) (Figura 3). Por otra parte, en los índices calculados (Tabla 3 ) no se encontraron diferencias significativas $(p>0.05)$ en ningún BA. El RAS indica el riesgo potencial del exceso de sodio sobre los elementos calcio y magnesio, los valores os- 


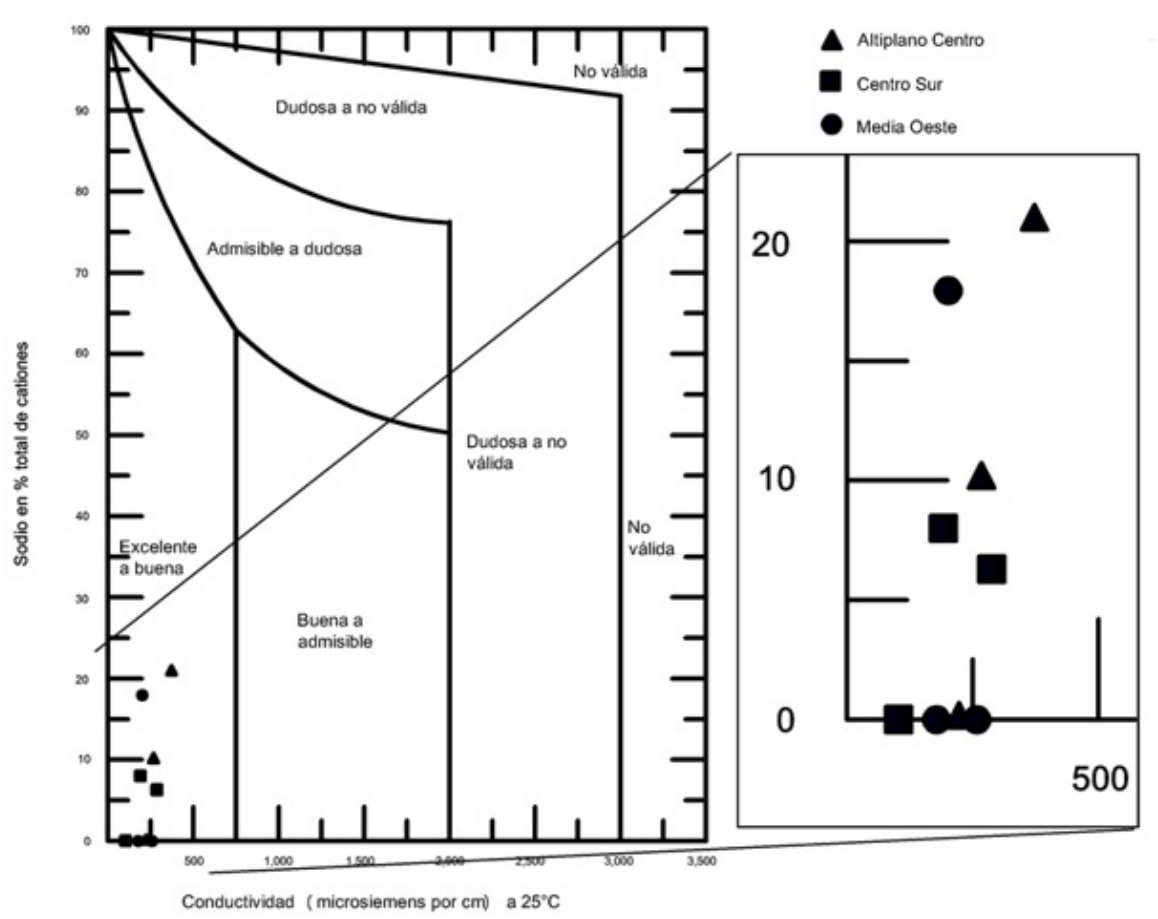

Figura 2. Clasificación Wilcox del agua de BA en tres microrregiones de San Luis Potosí.

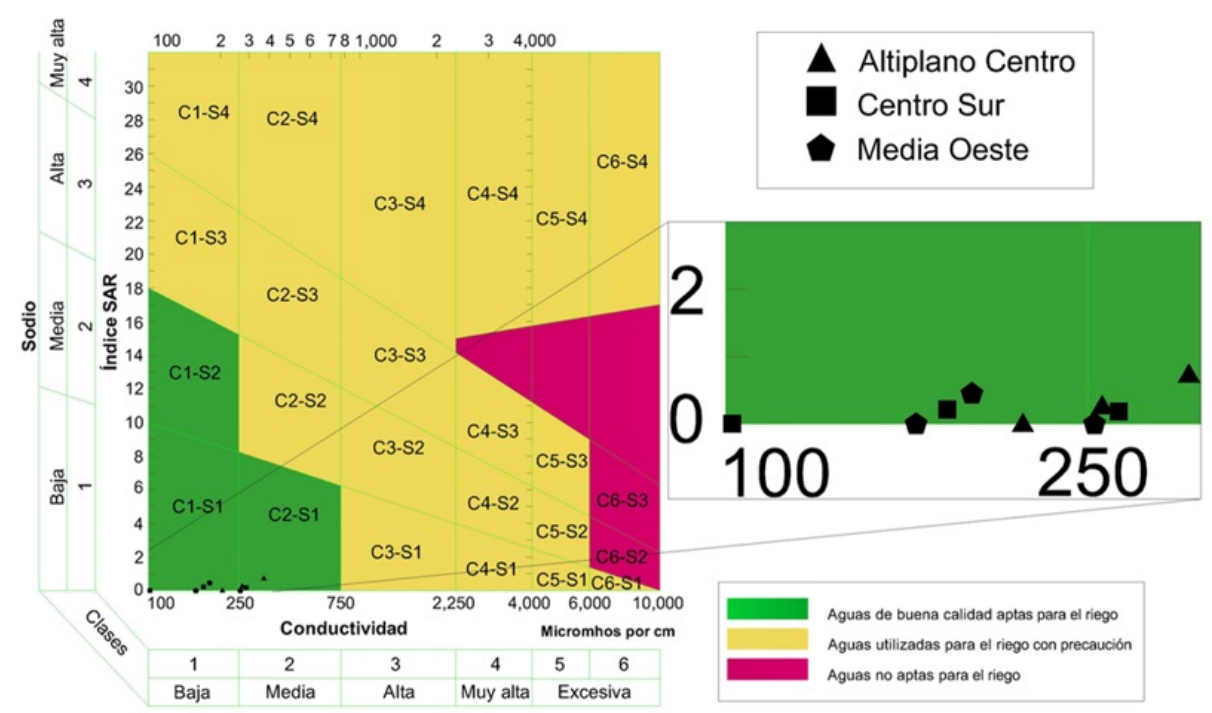

Figura 3. Calidad del agua de BA en tres microrregiones de San Luis Potosí de acuerdo a las Normas de Riverside.

cilaron entre 0.00 y 0.73 . Mediante el CSR se estima la peligrosidad del sodio cuando la concentración de carbonatos y bicarbonatos es mayor que la de calcio más magnesio, el rango obtenido para este índice fue de -0.70 a 0.60 meq $L^{-1}$. Otro parámetro para estimar la sodicidad es el PSP, el cual se refiere a la proporción de sodio sobre el total de cationes cuando hay precipitación de carbonatos de calcio y magne- 


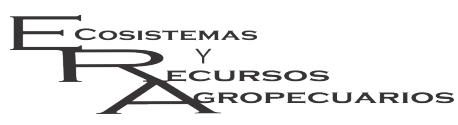

sio, así como sulfato de calcio, con valores de 0.0 a $63.5 \%$ mientras que el IP osciló entre 59.29 y $83.25 \%$.

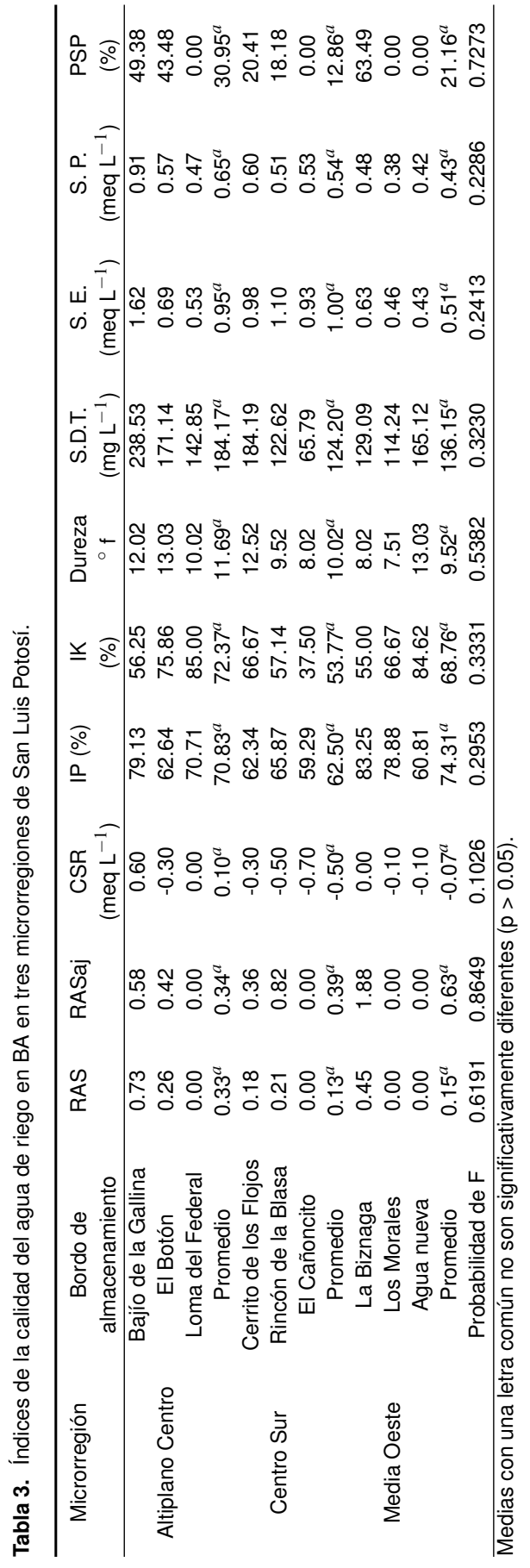

En lo que respecta al porcentaje de calcio sobre el total de cationes (IK) se encontraron valores entre 37.50 y $85.00 \%$. La cantidad de iones calcio y
Agua captada en bordos para agricultura

Ecosist. Recur. Agropec.

6(17):283-295,2019

magnesio solubles en el agua y dureza fue de 7.51 a $13.03^{\circ} \mathrm{fH}$. El RASaj tuvo valores entre 0.00 y 1.88 (Tabla 3). Para conocer el posible efecto de las sales disueltas del agua en la solución del suelo se estimaron los SDT, SE y SP. La concentración de SDT varió entre 65.79 y $238.53 \mathrm{mg} \mathrm{L}^{-1}$. La SE en el agua de los $B A$ fue de 0.43 a $1.62 \mathrm{meq}^{-1}$. Mientras que la SP osciló entre 0.38 y $0.91 \mathrm{meq} \mathrm{L}^{-1}$.

\section{DISCUSIÓN}

El pH del agua de lluvia captada en los BA fue alcalino, lo que coincide con Uba y Aghogho (2000) quienes encontraron una variación de pH entre 7.02 y 7.45 para agua captada en techos de aluminio, zinc, asbesto y paja; mientras que en la presente investigación el agua provino de reservorios a cielo abierto. Con respecto al pH, Lee et al. (2010) compararon el pH del agua de lluvia captada en tanques de PVC y una presa, encontrando que el agua de la presa presentó valores entre 7.5 a 8.3 , mientras que el pH del agua de lluvia osciló entre 4.3 y 6.0. Al respecto, Berndtsson et al. (2009) señalan que el incremento del $\mathrm{pH}$ en el agua de escorrentía (7.5) con relación al agua de lluvia indica una rápida neutralización de las deposiciones ácidas, lo que es un beneficio ambiental cuando la escorrentía descarga de forma directa en reservorios de agua natural. La alcalinidad del $\mathrm{pH}$ puede atribuirse a los carbonatos de calcio y magnesio aportados por la erosión eólica y lixiviados del agua de lluvia (Melidis et al. 2007) o por los que provienen de la erosión del suelo trasportados por los escurrimientos superficiales (Ali et al. 2004). Los valores de CE del agua almacenada en los BA de las tres microrregiones tuvo valores entre 103 y $373 \mu \mathrm{S}$ $\mathrm{cm}^{-1}$, los cuales de acuerdo con los criterios ecológicos CE-CCA-001/89 son bajos (SEDUE 1989). Al respecto Selala et al. (2018) reportan valores de CE de $61.4 \mu \mathrm{S} \mathrm{cm}^{-1}$ en el agua de una presa aguas abajo, valor superior a la CE del agua captada en techos de paja (18 $\left.\mu \mathrm{S} \mathrm{cm}^{-1}\right)$, lo que asociaron con el transporte lateral de sólidos por escorrentía superficial.

El orden de la abundancia de los aniones en las tres áreas de estudio fue similar $\left(\mathrm{HCO}_{3}^{-}>\mathrm{Cl}^{-}>\right.$ 
$\mathrm{SO}_{4}^{2-}$ ), lo que coincide con los resultados de RamosLeal et al. (2016) para aguas subterráneas de la región Centro de San Luis Potosí. Con respecto a los cationes, el orden fue similar para MCS y MMO $\left(\mathrm{Ca}^{2+}>\mathrm{K}^{+}>\mathrm{Na}^{+}>\mathrm{Mg}^{2+}\right)$ pero diferente para MAC $\left(\mathrm{Ca}^{2+}>\mathrm{K}^{+}>\mathrm{Mg}^{2+}>\mathrm{Na}^{+}\right)$. Lo que difiere de lo reportado por Ramos-Leal et al. (2016) quienes señalan el siguiente orden: $\mathrm{Ca}^{2+}>\mathrm{Mg}^{2+}>\mathrm{Na}^{+}>\mathrm{K}^{+}$. Mientras que Mirás et al. (2012) reportaron el orden siguiente: $\mathrm{Na}^{+}>\mathrm{Ca}^{2+}>\mathrm{K}^{+}>\mathrm{Mg}^{2+}$. La mayor concentración de bicarbonato en el agua de los $\mathrm{BA}$ puede ser producto del intercambio de $\mathrm{CO}_{2}$ atmosférico con el agua, el cual al ingresar al sistema cambia a $\mathrm{HCO}_{3}^{-}$ (Mahato et al. 2016). En los BA la concentración de $\mathrm{HCO}_{3}^{-}$varió entre 54.9 y 183.0 ppm, valores que son mayores a los reportados por Cobbina et al. (2013) y Mahato et al. (2016) quienes reportan concentraciones entre 2.4 y 43.9 ppm, lo que se puede asociar con aguas alcalinas.

Para el $\mathrm{Cl}^{-}$se tuvo una concentración de 16.15 ppm, valores que son similares a lo reportado por Lee et al. (2010) e indica que las plantas no son afectadas por toxicidad de cloruro (Ayers y Westcot 1994). Mientras que Sazakli et al. (2007), Cobbina et al. (2013) y Mahato et al. (2016) reportan una menor concentración de $\mathrm{Cl}^{-}(7.00,3.56$ y 3.25 ppm, respectivamente). Lo anterior puede deberse a que el $\mathrm{Cl}^{-}$ del agua de lluvia posiblemente provenga de actividades antropogénicas como la incineración de desechos (Younos et al. 1998). La concentración de $\mathrm{SO}_{4}^{2-}$ promedio (8 ppm) fue baja, pero es similar a la reportada por Sazakli et al. (2007), Lee et al. (2010) y Mahato et al. (2016). Mientras que la baja concentración de sulfato puede indicar una moderada o insignificante transformación química de dióxido de azufre en la atmósfera por actividad del hombre (Cobbina et al. 2013).

El catión más abundante en el agua de los BA en las tres microrregiones fue el $\mathrm{Ca}^{2+}$ con concentración promedio de 30.3 ppm, valor que es similar al obtenido por Lee et al. (2010), pero dos veces más alto que los valores reportados por Sazakli et al. (2007). Debido a que el mayor volumen de agua en los BA proviene de escurrimientos superficiales, la mayor cantidad de calcio puede atribuirse al arras- tre de partículas de suelo en forma de carbonatos (Ali et al. 2004). Por otra parte, la concentración de $\mathrm{K}^{+}$fue mayor que la registrada por Sazakli et al. (2007) y Mahato et al. (2016). Al respecto, Simmonds et al. (2013) reportan que este ion se desplaza de las partes altas de la cuenca por flujo de masas, disminuyendo la disponibilidad de este elemento para las plantas. Con respecto al $\mathrm{Na}^{+}$, se obtuvo una concentración promedio de $4.9 \mathrm{ppm}$ la cual fue dos veces superior a la reportada por Selala et al. (2018). Una alta concentración de sodio en el agua de riego puede provocar que se adsorba en los sitios de intercambio catiónico del suelo afectando la estructura del suelo y la infiltración (Warrence et al. 2003).

La concentración de $\mathrm{Mg}^{2+}$ en BA (6.9 ppm) fue inferior a la reportada (16 ppm) en agua de lluvia captada en reservorios (Lee et al. 2010). Sobre lo mismo Mirás et al. (2012) encontraron en agua de escorrentía, concentraciones de cationes entre 8.4 a 11.2 ppm para $\mathrm{Ca}^{2+}, 3.1$ a 4.3 para $\mathrm{Mg}^{2+}, 3.9$ a $13.2 \mathrm{ppm} \mathrm{K}^{+}$y 14.3 a 20.9 ppm para $\mathrm{Na}^{+}$. Esto indica que los suelos son someros y de baja infiltración por lo que las concentraciones determinadas en el agua provienen de la disolución del material constituyente del suelo desplazado por la escorrentía superficial (Mason et al. 1999, Berndtsson et al. 2009).

Desde un punto de vista agrícola, la calidad del agua se refiere al tipo y la cantidad de sales presentes y su efecto sobre el suelo y el desarrollo de los cultivos (Cortés-Jiménez et al. 2009). El 100\% de las aguas de los BA fue de buena a excelente calidad de acuerdo con la Clasificación de Wilcox. Mientras que las Normas de Riverside las clasificaron como aptas para el riego $\left(55 \% \mathrm{C}_{1} \mathrm{~S}_{1}\right.$ y $\left.45 \% \mathrm{C}_{2} \mathrm{~S}_{1}\right)$. Al respecto, Blasco y De la Rubia (1973) señalan que la irrigación con aguas $C_{1}$ se pueden tener problemas en suelos de baja permeabilidad. Mientras que con las $C_{2}$, puede requerirse el lavado de sales por lixiviación o el uso de cultivos tolerantes a la salinidad y las $S_{1}$, pueden generar problemas en cultivos sensibles al sodio. De acuerdo con los criterios de Ayers y Westcot (1994) el índice RAS y la CE indican que el agua de los BA Los Morales (MMO), El Cañoncito y Rincón de la Blasa (MCS) afectan el crecimiento de las plantas, debido a que los valores de CE fueron 
inferiores a $0.2 \mathrm{dS} \mathrm{m}^{-1}$ lo que favorece la lixiviación de minerales del suelo como el $\mathrm{Ca}^{2+}$ y se afecta la estructura, permeabilidad y aireación (Saleem et al. 2015). Por lo que la velocidad de infiltración del agua en el suelo se afectaría en las áreas de los BA Los Morales (MMO), El Cañoncito y Rincón de la Blasa (MCS), para el BA MCS fueron reportados resultados similares por Reyna et al. (1980) y para el BA MMO por Villanueva y Hernández (2001). Mientras que para el resto de los BA se podría tener una infiltración de ligera a moderada.

De acuerdo con el CSR el $68 \%$ de los BA tuvieron problemas de carbonato sódico residual, mientras que el $32 \%$ restante de los BA no tienen problemas debido a que el CSR tiene signo positivo, lo que indica que el calcio y magnesio se precipitan en forma de carbonatos. Por lo que en la solución sólo hay sales de sodio (Medina et al. 2016), las cuales pueden afectar las propiedades físicas del suelo (Naidu y Rengasamy 1993). Por lo que se recomienda evaluar de forma periódica la velocidad de infiltración del agua y el pH del suelo (Stevens 1994). Al respecto, Villanueva y Hernández (2001) reportan un índice CSR peligroso para el agua de pozos profundos de la MMO. La clasificación realizada por García (2015) para el PSP indica que sólo en La Biznaga se pueden generar acumulaciones de sodio que participen en la compactación del suelo (Rodríguez et al. 2008).

Para el índice de permeabilidad (IP) se utilizó el criterio propuesto por Balmaseda et al. (2006), que clasificó las aguas de los BA Bajío de la Gallina (MAC), La Biznaga y Los Morales (MMO) como excelentes (IP > 75\%), mientras que el resto de los BA se clasificaron como buenos (PI: 25 - 75\%). Resultados que concuerdan con los encontrados por Ramos-Leal et al. (2016), pero se difiere con Gonzáles-Acevedo et al. (2016) quienes clasifican al agua como no recomendable. Debido a que el IP mide el riesgo del sodio sobre la permeabilidad del suelo por el uso del agua de riego a largo plazo (Brindha y Elango 2011) y en función del tipo de suelo y la calidad del agua de riego (González-Acevedo et al. 2016), las aguas de los BA en las tres microrregiones no tendrían un efecto negativo sobre el suelo a largo plazo. So- bre el porcentaje de calcio entre el total de cationes (IK), Camacho y Fernández (2008) mencionan que si las aguas tienen un IK mayor a $35 \%$ son aptas para irrigación, por lo que el $100 \%$ de las aguas de los BA fueron aptas para irrigación. Lo que difiere de Ramos-Leal et al. (2016) quienes reportan valores de IK inapropiados para irrigación. Mientras que González-Acevedo et al. (2016) reportan que el $100 \%$ de las aguas subterráneas y $75 \%$ de las aguas superficiales son inapropiadas para el riego.

Para evitar la subestimación del RAS se estimó el RASaj. Los valores promedio de RASaj y RAS (0.865 y 0.619 , respectivamente) indican una relación 1:1 entre estos índices. Al respecto Levy et al. (2014) sugirieron que hasta un valor de 3.5 de RAS de cualquier muestra se obtendrá un valor similar para el RAS no ajustado y el RASaj. El 100\% del agua captada en los BA presenta una tendencia a precipitar el $\mathrm{CaCO}_{3}$ de la solución del suelo, lo que tiene implicaciones en la solubilidad de fósforo, hierro y zinc, por lo que se reduce la absorción de estos nutrimentos por la planta. De acuerdo con la clasificación propuesta por Orta (2006) en todos los bordos el agua es dulce $\left(7 \mathrm{a} 14^{\circ} \mathrm{fH}\right)$ resultados que contrastan con el agua de pozos profundos, donde se ha encontrado que el $70 \%$ de las aguas son duras (Sarabia et al. 2011). En tanto que los índices de SDT, SE y SP, indican que el $100 \%$ de las aguas de bordos no tienen restricciones para uso en riego (Castellanos et al. 2000). Mientras que Ramos-Leal et al. (2016) reportan aguas con restricción leve a moderada y sin grado de restricción por SP.

Un factor importante que determina la aptitud de agua para riego es la tolerancia de los cultivos a la salinidad (Aragües 2011). En este sentido Ayers y Westcot (1994) y Kotuby-Amacher et al. (2000), han determinado los umbrales de tolerancia a la salinidad del maíz (1.8 dS $\left.\mathrm{m}^{-1}\right)$, avena (2.6 dS $\left.\mathrm{m}^{-1}\right)$, sorgo $\left(6.8 \mathrm{dS} \mathrm{m}^{-1}\right)$ y cebada $\left(8.0 \mathrm{dS} \mathrm{m}^{-1}\right)$, a partir del cual el rendimiento relativo disminuye con el aumento de la CE del extracto del suelo. De acuerdo con los valores estimados de CEe $\left(<0.6 \mathrm{dS} \mathrm{m}^{-1}\right)$ a partir de la CE del agua de los BA y los umbrales de tolerancia de dichos cultivos (Figura 4), el rendimiento relativo de estos no se afecta irrigándolos con agua de los 


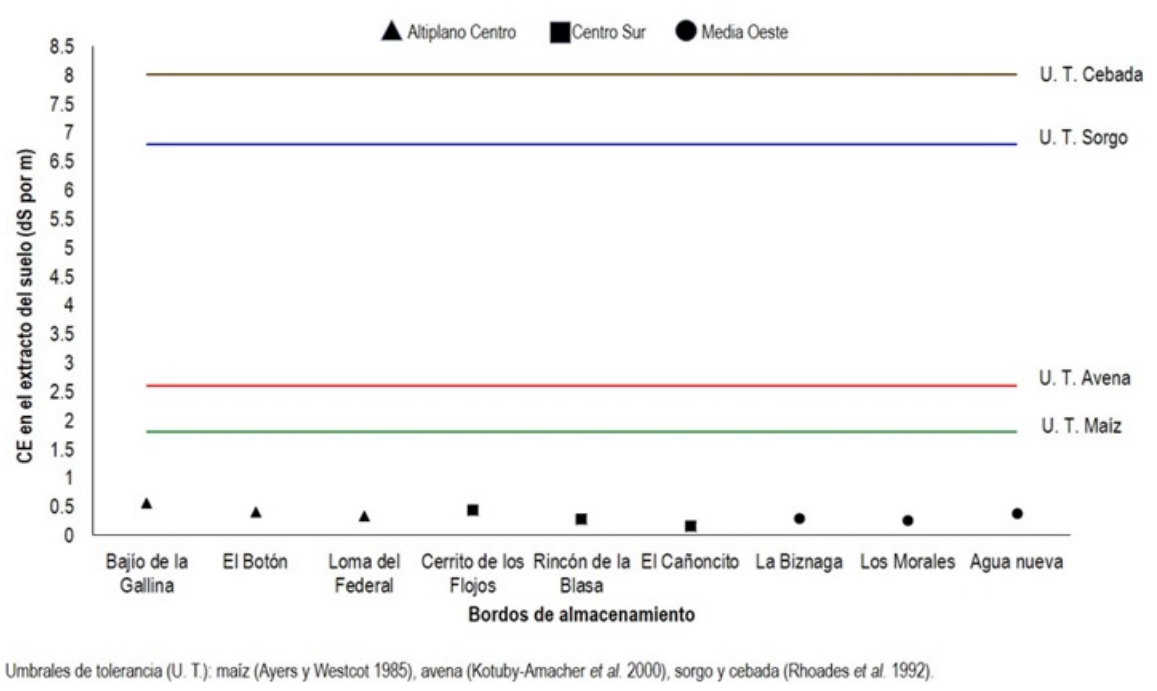

Figura 4. Valores estimados de CE en el extracto del suelo a partir de la CE del agua de BA y umbrales de tolerancia de cultivos forrajeros.

BA, debido a que la CEe está por debajo del umbral de tolerancia de los cuatro cultivos. Considerando la buena calidad del agua de los BA y los pequeños volúmenes de agua almacenada, es recomendable su uso en la irrigación de superficies menores a 1 ha.

\section{CONCLUSIONES}

El agua de los BA es de calidad buena a excelente para uso agrícola y según las Normas de Riverside, es apta para el riego, con problemas en suelos de muy baja permeabilidad $\left(C_{1}\right)$, sembrando cultivos sensibles a la salinidad $\left(\mathrm{C}_{2}\right)$ y sodio $\left(\mathrm{S}_{1}\right)$. Los índices IP, IK, SDT, SE y SP indican que no hay restricción para el uso del agua de los $\mathrm{BA}$ en el riego agrícola. Mientras que el RAS y la CE indican ligero a severo efecto en la infiltración de los BA. De acuerdo al CSR, el $33 \%$ de los BA tienen un ligero riesgo de sodicidad y según el índice PSP este riego está presente en el $11 \%$ de los casos. El agua de los BA es dulce con tendencia a precipitar el carbonato de calcio, y buena calidad para riego. Es necesario evaluar la aptitud del agua en conjunto con las características edafo-climáticas del sitio para definir las prácticas de manejo requeridas para incrementar la productividad.

\section{AGRADECIMIENTOS}

Al Consejo Nacional de Ciencia y Tecnología (CONACYT) por la beca a Luz Andrea Loera Alvarado para realizar sus estudios de Maestría en Ciencias.

\section{LITERATURA CITADA}

Akhtar M, Hassan FU, Ahmed M, Hayat R, Stöckle CO (2016) Is rainwater harvesting an option for designing sustainable cropping patterns for rainfed agriculture? Land degradation \& development 27: 630-640.

Ali K, Momin GA, Tiwari S, Safai PD, Chate DM, Rao PSP (2004) Fog and precipitation chemistry at Delhi, North India. Atmospheric Environment 38: 4215-4222.

Aragües LR (2011) Agricultura y calidad de aguas a nivel fuente y sumidero. Riegos y Drenajes XXI 182: 24-33.

Ayers RS, Westcot DW (1994) Water quality for agriculture. FAO Irrigation and Drainage Paper 29 Rev.1. Rome, Italy. 130p. 
Balmaseda C, Ponce de León D, Martín NJ, Vargas H (2006) Compendio de Suelo. Facultad de Agronomía, Departamento de Riego, Drenaje y Ciencias del Suelo. Universidad Agraria de la Habana, Fructuoso Rodríguez Pérez. 288p.

Berndtsson JC, Bengtsson L, Jinno K (2009) Runoff water quality from intensive and extensive vegetated roofs. Ecological Engineering 35: 369-380.

Blasco F, De la Rubia J (1973) Guía para clasificar las aguas en relación con su calidad para el riego. Instituto para la Reforma y Desarrollo Agrario. Madrid, España. 322p.

Bower CA, Wilcox LV (1965) Precipitation and solution of calcium carbonate in irrigation operations. Soil Science Proceedings 29: 93-94.

Brindha K, Elango L (2011) Hydrochemical characteristics of groundwater for domestic and irrigation purposes in Madhuranthakam, Tamil Nadu, India. Earth Sciences Research Journal 15: 101-108.

Camacho FF, Fernández EJ (2008) Manual práctico de fertirrigación en riego por goteo. Ediciones Agrotécnicas. Madrid, España. 169p.

Castellanos JZ, Uvalle BJX, Aguilar SA (2000) Manual de interpretación de análisis de suelos, aguas agrícolas, plantas y ECP. 2da edición. INTAGRI. México. 201p.

Cobbina SJ, Michael K, Salifu L, Duwiejua AB (2013) Rainwater quality assessment in the Tamale Municipality. International Journal of Scientific \& Technology Research 2: 1-10.

CONAGUA (2018) Numeragua México 2018. Comisión Nacional del Agua. sina.conagua.gob.mx/publicaciones/ Numeragua_2018.pdf. Fecha de consulta: 20 de febrero de 2019.

Cortés-Jiménez JJM, Troyo-Diéguez E, Murillo-Amador B, García-Hernández JL, Garatuza-Payán J, et al. (2009) Índices de calidad del agua del acuífero del Valle del Yaqui, Sonora. Terra Latinoamericana 27: 133-141.

Doneen LD (1964) Notes on water quality in agriculture. Department of Water Science and Engineering, University of California. Davis, California. 400p.

Doneen LD (1975) Water quality for irrigated agriculture. In: Poljakoff-Mayber A, Gale J (eds). Plant in saline environments ecological studies. Springer Verlag. USA. pp: 56-76.

Eaton FM (1950) Significance of carbonates in irrigation waters. Soil Science 69: 123-133.

FAO (2013) Afrontar la escasez de agua, un marco de acción para la agricultura y la seguridad alimentaria. Organización de las Naciones Unidas para la Alimentación y la Agricultura. Roma, Italia. 97p.

García HY (2015) Calidad del agua con fines de riego. Revista Digital de Medio Ambiente 35: 1-12.

García OA (2012) Criterios modernos para evaluación de la calidad del agua de riego (Primera parte). Informaciones Agronómicas de Hispanoamérica 6: 27-34.

González-Acevedo ZI, Padilla-Reyes DA, Ramos-Leal JA (2016) Quality assessment of irrigation water related to soil salinization in Tierra Nueva, San Luis Potosi, Mexico. Revista Mexicana de Ciencias Geológicas 33: 271-285.

Helmreich B, Horn H (2009) Opportunities in rainwater harvesting. Desalination 248: 118-124.

INEGI (2006) Carta topográfica de precipitación media anual. Mapa Digital de México. Instituto Nacional de Estadística y Geografía. México. http://gaia.inegi.org.mx/mdm6/?v=Bgf0Ojl0Ljc5ODMyLGxvbjotOTg uMzl1MjAsejoyLGw6YzExMXNIcnZpY2lvc3x0YzExMXNIcnZpY2lvcw==. Fecha de consulta: 06 de marzo de 2018. 
INEGI (2011) Carta topográfica de uso del suelo y vegetación. Mapa Digital de México. Instituto Nacional de Estadística y Geografía. México. http://gaia.inegi.org.mx/mdm6/?v=bGF0OjlOLjc5ODMyLGxvbjotOT guMzl1MjAsejoyLGw6YzExMXNIcnZpY2Ivc3x0YzExMXNIcnZpY2lvcw==. Fecha de consulta: 06 de marzo de 2018.

INEGI (2016) Conjunto de datos vectoriales de información topográfica escala 1: 50000 serie III. Instituto Nacional de Estadística y Geografía. México. http://www.beta.inegi.org.mx/app/mapas//?t=0150 0010000000 00\&tg=999\&esc=50000. Fecha de consulta: 10 de enero de 2016.

Kahinda JM, Rockstrom J, Taigbenu AE, Dimes J (2007) Rainwater harvesting to enhance water productivity of rainfed agriculture in the semi-arid Zimbabwe. Physics and Chemistry of the Earth 32: 1068-1073.

Kelly WP (1951) Alkali soils - their formation, proprieties and reclamation. 3rd ed. Reinhold, New York. 92p.

Klaus-Dieter B (2008) Case study "Rainwater harvesting". In agricultural technologies for developing countries. European Parliament. Science Technology Options Assessment (STOA).Tübingen, Germany. 49p.

Kotuby-Amacher J, Koening R, Kitchen B (2000) Salinity and plant tolerance. Electronic publishing. AG-SO-03. https://digitalcommons.usu.edu/cgi/viewcontent.cgi?referer=https://www.google.com/\&httpsredir=1\&article= 1042\&context=extension_histall . Fecha de consulta: 01 de mayo de 2018.

Lee JY, Yang JS, Han M, Choi J (2010) Comparison of the microbiological and chemical characterization of harvested rainwater and reservoir water as alternative water resources. Science of the Total Environment 408: 896-905.

Levy GJ, Fine P, Goldstein D, Azenkot A, Zilberman A, Chazan A, Grinhut T (2014) Long term irrigation with treated wastewater (TWW) and soil sodification. Biosystems Engineering 128: 4-10.

Mahato MK, Singh PK, Singh AK, Tiwari AK (2016) Assessment of major ionic compositions and anthropogenic influences in the rainwater over a coal mining environment of Damodar River basin, India. Pollution 2: 461-474.

Mason Y, Ammann AA, Ulrich A, Sigg L (1999) Behavior of heavy metals, nutrients and major components during roof runoff infiltration. Environmental Science \& Technology 33: 1588-1597.

Medina VEK, Mancilla VOR, Larios MM, Guevara GRD, Olguín LJL, Barreto GOA (2016) Calidad del agua para riego y suelos agrícolas en Tuxcauesco, Jalisco. Idesia. 34: 51-59.

Melidis P, Akratos CS, Tsihrintzis VA, Trikilidou E (2007) Characterization of rain and roof drainage water quality in Xanthi, Greece. Environmental monitoring and assessment 127: 15-27.

Mirás AJM; Sande FP, Bertol I, Paz GA (2012) Crop residue effects on calcium, magnesium, potassium, and sodium runoff losses from a soil prone to crusting. Communications in Soil Science and Plant Analysis 43: 315-323.

Naidu R, Rengasamy P (1993) Ion interaction and constraints to plant nutrition in Australian sodic soil. Australian Journal Soil Science 31: 801-819.

Orta SR (2006) La empresa de jardinería y paisajismo: mantenimiento y conservación de espacios verdes. 3da edición. Mundi-Prensa. España. 544p.

Oweis T, Hachum A (2006) Water harvesting and supplemental irrigation for improved water productivity of dry farming systems in West Asia and North Africa. Agricultural Water Management 80: 57-73.

Palacios VO, Aceves NE (1970) Instructivo para el muestreo, registro de datos e interpretación de la calidad del agua para riego agrícola. Chapingo. Colegio de Postgraduados. México. 49p. 
Pimentel D, Berger B, Filiberto D, Newton M, Wolfe B, Karabinakis E, et al. (2004) Water resources: Agricultural and environmental Issues. BioScience 54: 909-918.

Ramos-Leal JA, López-Álvarez B, Santacruz-De León G, Almanza-Tovar O, Morán-Ramírez J, Padilla-Reyes DA, et al. (2016) Quality indices of groundwater for agricultural use in the region of Tierra Nueva, San Luis Potosi, Mexico. Arabian Journal of Geosciences 9: 736. Doi: 10.1007/s12517-016-2758-2

Reyna TT, Villegas SM, Gómez TRA (1980) Estudio edáfico climático del Ejido El Rosario y los potreros El Chivato y Granadillas, en Villa de Reyes, San Luis Potosí. Investigaciones Geográficas 10: 181-207.

Richards LA (1954) Diagnosis and improvement of saline and alkali soils. US Department of Agriculture Handbook 60. Washington, USA. 166p.

Rodríguez M, D’Urso C, Rodríguez G, Sales A (2008) Evaluación de la calidad de aguas para riego de la cuenca del Río Calera, Tucumán, Argentina. Ciencia 3: 15-28.

Rosegrant M, Cai X, Cline S, Nakagawa N (2002) The role of rained agriculture in the future of global food production. International Food Policy Research Institute. No. 90. Washington, USA. 127p.

Saleem M, Iqbal J, Shah MH (2015) Assessment of water quality for drinking/irrigation purpose from Mangla dam, Pakistan. Geochemistry, Exploration, Environment, Analysis 16: 137-145.

Sarabia MIF, Cisneros AR, Aceves AJ, Durán GHM, Castro LJ (2011) Calidad del agua de riego en suelos agrícolas y cultivos del Valle de San Luis Potosí, México. Revista Internacional de Contaminación Ambiental 27: 103-113.

Sazakli E, Alexopoulos A, Leotsinidis M (2007) Rainwater harvesting, quality assessment and utilization in Kefalonia Island, Greece. Water Research 41: 2039 - 2047.

SEDUCOP (2012) Plan estatal de desarrollo urbano de San Luis Potosí 2012 - 2030. Secretaría de Desarrollo Urbano, Comunicaciones y Obras Públicas. San Luis Potosí, México. 564p.

SEDUE (1989) Criterios ecológicos de calidad del agua CE-CCA-001/89. Secretaría de Desarrollo Urbano y Ecología. México. 18p.

Selala MS, Thenga H, Jewitt GPW, Chaplot V (2018) Comparison of the chemical quality of rainwater harvested from roof and surface run-off systems. Water SA 44: 223-231.

SEMARNAT (2015) Inventario estatal forestal y de suelos - San Luis Potosí 2014. Secretaría de Medio Ambiente y Recursos Naturales. 1 edición. México. 190p.

Simmonds MB, Plant RE, Peña-Barragán JM, Van Kessel C, Hill J, Linquist BA (2013) Underlying causes of yield spatial variability and potential for precision management in rice systems. Precision Agriculture 14: 512-540.

Sparks DL (1995) Environmental Soil Chemistry, Academic Press, Inc. San Diego, California. 269p.

SSA (2005) Norma Oficial Mexicana NOM-230-SSA1-2002, Salud ambiental. Agua para uso y consumo humano, requisitos sanitarios que se deben cumplir en los sistemas de abastecimiento públicos y privados durante el manejo del agua. Procedimientos sanitarios para el muestreo. Secretaría de Salud. Diario Oficial de la Federación. México. http://www.salud.gob.mx/unidades/cdi/nom/230ssa102.html. Fecha de consulta: 19 de marzo de 2016.

Stevens RG (1994) Water quality and treatment considerations. In: Williams KE, Ley TW (eds). Tree fruit irrigation: A comprehensive manual of deciduous tree fruit irrigation needs. Good Fruit Publishing. Washington State Fruit Commission. Yakima. WA, USA. pp: 115-125. 
Uba BN, Aghogho O (2000) Rainwater quality from different roof catchments in the Port Harcourt district, Rivers State, Nigeria. Journal of Water Supply: Research and Technology-AQUA 49: 281-288.

Vásquez VA (2000) Manejo de cuencas Alto Andinas. Tomo I. Ed. Firmart. Universidad Nacional Agraria La Molina. Lima-Perú. 516p.

Villanueva DJ, Hernández RA (2001) Calidad de agua en tres áreas de San Luis Potosí y su efecto en la producción de cultivos. Instituto Nacional de Investigaciones Forestales, Agrícolas y Pecuarias (INIFAP). Folleto técnico Número 13. San Luis Potosí, México. 32p.

Warrence KJ, Pearson KE, Bauder JW (2003) The basics of salinity and sodicity effects on soil physical properties. Land Resources and Environmental Sciences Department. Montana State University. Bozeman, USA. 29p.

Wilcox LV (1948) The quality of water for irrigation. Department of Agriculture. Tech Bulletin 962. Washington. 40p.

Younos TM, Bohdan R, Anderson E, Ramsey KA, Cook N, Ross B, et al. (1998) Evaluation of rooftop rainfall collection-cistern storage systems in Southwest Virginia. Blacksburg. 42p. 
